\title{
Effects of Repaglinide Versus Glimepiride on 1,5-Anhydroglucutol and Glycated Hemoglobin Levels in Japanese Patients With Type 2 Diabetes
}

\author{
Hodaka Yamada ${ }^{\text {a, b }}$, Masafumi Kakei ${ }^{\text {a }}$ Kazuo Hara ${ }^{\text {a }}$
}

\begin{abstract}
Background: Postprandial hyperglycemia is a well-known risk factor for cardiovascular disease. We prospectively examined the effects of repaglinide on postprandial hyperglycemia in patients with type 2 diabetes.
\end{abstract}

Methods: During this 24-week, single-arm, prospective study, we enrolled 10 patients with type 2 diabetes who were previously being administered glimepiride $(0.5$ or $1 \mathrm{mg} /$ day $)$ and switched it with repaglinide ( 0.75 or $1.5 \mathrm{mg}$ /day). Changes in their metabolic parameters were evaluated at the end of the study period.

Results: After replacing glimepiride with repaglinide, increases were observed in 1,5-anhydroglucitol levels (baseline, $5.46 \pm 1.96$ versus 24 weeks, $9.15 \pm 4.48 \mu \mathrm{g} / \mathrm{mL}, \mathrm{P}=0.004$ ) but not in glycated hemoglobin levels (baseline, $7.7 \pm 0.5$ versus 24 weeks, $7.4 \pm 0.6 \%, \mathrm{P}=$ $0.100)$. Body weight remained unchanged.

Conclusion: Compared with glimepiride, repaglinide improved 1,5-anhydroglucitol levels but had no effect on glycated hemoglobin. This suggests that repaglinide is a useful option for treating postprandial hyperglycemia.

Keywords: Postprandial hyperglycemia; Type 2 diabetes; 1,5-anhydroglucitol; Glinide; Sulfonylurea

\section{Introduction}

Several epidemiological studies have identified postprandial hyperglycemia as a well-known risk factor for cardiovascular

Manuscript submitted September 11, 2018, accepted September 27, 2018

a Department of Medicine, Division of Endocrinology and Metabolism, Jichi Medical University Saitama Medical Center, 1-847 Amanuma-cho, Omiya-ku, Saitama 330-8503, Japan

${ }^{b}$ Corresponding Author: Hodaka Yamada, Department of Medicine, Jichi Medical University Saitama Medical Center, 1-847 Amanuma-cho, Omiyaku, Saitama 330-8503, Japan. Email: hyamada0510@jichi.ac.jp

doi: https://doi.org/10.14740/jocmr3618w diseases [1-3]. Specifically, postprandial hyperglycemia generates oxidative stress and consequently causes endothelial cell injury and dysfunction, which is important to both initiation and progression of atherosclerosis $[4,5]$.

Previous clinical studies involving continuous glucose monitoring (CGM) revealed that compared with fasting glucose level, postprandial glucose level was a greater contributor to $\mathrm{HbA1c}$ among patients with glycated hemoglobin (HbAlc) levels $<8 \%[6,7]$. Further studies identified 1,5-anhydroglucitol $(1,5-\mathrm{AG})$ as a useful biomarker for postprandial hyperglycemia $[8,9]$ and for the efficacy of antihyperglycemic agents used to improve postprandial hyperglycemia [10]. Diabetes and its complications affect the anti-diabetic agent selection, for example, renal impairment, liver function, risks of hypoglycemia, and macro- or microangiopathy. Glinides are antihyperglycemic agents that enhance early-phase postprandial insulin secretion and improve postprandial hyperglycemia. However, few reports have evaluated the efficacy of repaglinide using 1,5-AG levels. Repaglinide, a member of the glinide class, is metabolized in the liver (CYP3A4 and CYP2C8) and excreted through the bile [11]. The major metabolites of repaglinide have no glucose-lowering effect [11]. Repaglinide is useful for the management of postprandial hyperglycemia. Therefore, in the present study, 1,5-AG was used to prospectively examine the effects of repaglinide in patients with type 2 diabetes who switched from glimepiride, a major sulfonylurea drug, to repaglinide.

\section{Patients and Methods}

\section{Patients and study design}

The current study was approved by the Ethics Committee of the Jichi Medical University, Japan (No. 12-51); written informed consent in accordance with the Declaration of Helsinki was obtained from all patients. This 24 -week, single-arm, prospective study was conducted between January 2013 and January 2014. The following inclusion criteria were considered for enrollment in this study: 1 ) patients aged $>20$ years and having type 2 diabetes and 2) patients prescribed treatment comprising glimepiride $(0.5$ or $1 \mathrm{mg} /$ day). Additionally, the following criteria were considered for exclusion from the study: 1) diabetic ketoacidosis or hyperosmolar hyperglycemic syndrome, 2) infectious diseases, 3) any malignancy during treatment, 4) worsening 


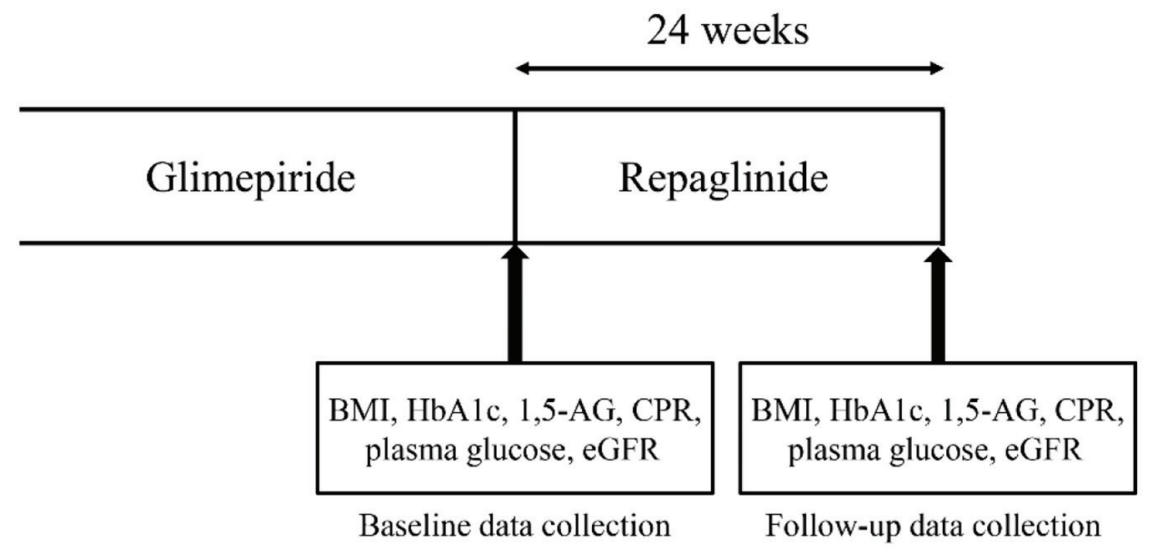

Figure 1. Study protocol. Japanese patients with type 2 diabetes were enrolled and followed-up for 24 weeks after switching from glimepiride to repaglinide. Clinical metabolic parameters were measured and recorded at weeks 0 (baseline) and 24.

diabetes or progressive diabetic complication (e.g. progressive retinopathy and nephropathy), 5) pregnancy, 6) acute coronary syndrome within 8 weeks prior to study enrollment and 7) recent changes in anti-diabetic drug therapy (within 8 weeks).

The study protocol is depicted in Figure 1. After recruitment, the body mass index (BMI), HbAlc level, random plasma glucose level, serum C-peptide immunoreactivity (CPR), 1,5-AG level and estimated glomerular filtration rate (eGFR) at baseline were determined for each patient. Subsequently, the ongoing glimepiride regimen was switched with repaglinide ( 0.75 or $1.5 \mathrm{mg}$ /day). The patients' metabolic parameters were recorded at the end of the study period. No antihyperglycemic agents were changed during the study period. Dieticians instructed all patients to follow diet therapy before the commencement of this study in accordance with Japanese Clinical Practice Guideline for Diabetes [12]. The primary and secondary study endpoints were the changes in the 1,5-AG and HbA1c levels, respectively.

\section{Data collection}

CPR was measured using an enzyme immunoassay (TOHSO, Tokyo, Japan). The serum levels of 1,5-AG were measured by SRL Inc. (Tokyo, Japan). Renal function was determined by calculating eGFR; this calculation was based on the modification of the renal disease equation, which was revised by the Japanese Society of Nephrology for the Japanese population. The equation is as follows: eGFR $\left(\mathrm{mL} / \mathrm{min} / 1.73 \mathrm{~m}^{2}\right)=194 \times$ serum creatinine $(\mathrm{mg} / \mathrm{dL})^{-1.094} \times$ age $^{-0.287} \times 0.739$ (if female) [13]. Delta $(\Delta)$ 1,5-AG was calculated the following equation: $\Delta 1,5-\mathrm{AG}=$ after switching $1,5-\mathrm{AG}$ - baseline 1,5-AG.

\section{Statistical analysis}

Data are expressed as mean \pm standard deviation, and skewed variables are expressed as medians with interquartile ranges. Paired $t$-test was used to compare the clinical parameters before and after switching to repaglinide. Pearson's correlation coefficient was used to assess the correlations between the baseline 1,5-AG level and $\Delta 1,5-\mathrm{AG}$. All analyses were performed using EZR (Jichi Medical University Saitama Medical Center, Saitama, Japan), a graphical user interface for R (v.2.13.0; The R Foundation for Statistical Computing, Vienna, Austria) and a modified version of the R commander (v.1.6-3), which was designed to add statistical functions frequently used in biostatistics research [14]. $\mathrm{P}<0.05$ was considered statistically significant.

\section{Results}

Initially, 14 eligible patients were identified; however, one patient was subsequently excluded for worsening diabetes, two for poor adherence to drug therapy and one more for discontinuing hospital visits (Fig. 2). Finally, total 10 patients were enrolled in this study, two and eight of whom had been prescribed glimepiride ( 0.5 and $1 \mathrm{mg} /$ day, respectively). The baseline clinical characteristics of all study participants and the other administered antihyperglycemic agents are presented in Table 1. Three patients were administered angiotensin II receptor blocker for hypertension. No patient took drugs that interacted with repaglinide.

Regarding the switch from glimepiride, two and eight patients were administered repaglinide (two patients, $1.5 \mathrm{mg}$ / day; eight patients, $0.75 \mathrm{mg} /$ day) during the study period. Following this drug switch, no changes were observed in the HbA1c levels (secondary endpoint) and body weight from the baseline to the end of the 24-week study period; however, the 1,5-AG levels (primary endpoint) significantly improved, and the baseline 1.5-AG and $\Delta 1.5-\mathrm{AG}$ exhibited a significant positive correlation $(\mathrm{r}=0.63, \mathrm{P}=0.049$; Fig. 3 ). No severe hypoglycemic events or other adverse events were observed throughout the study period.

\section{Discussion}

The serum 1,5-AG levels in patients with type 2 diabetes im- 


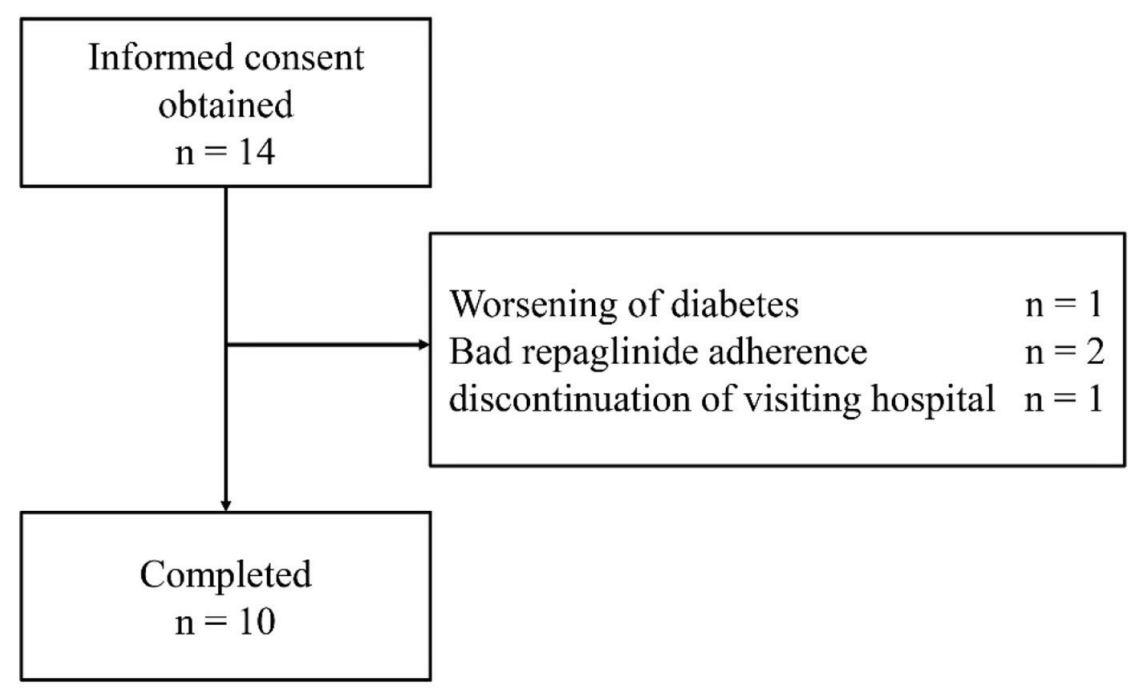

Figure 2. Flow chart of patient enrollment throughout the trial.

proved after glimepiride was switched to repaglinide; however, no concomitant changes were observed in the HbAlc levels. Even if the HbA1c level remains stable, a reduced serum 1,5-AG level reflects the occurrence of glycemic excursions, including postprandial hyperglycemia [8]. This result suggests that when compared with glimepiride, repaglinide suppresses postprandial hyperglycemia more efficiently.

Interestingly, a previous study reported that compared

Table 1. Baseline Characteristics of Patients Enrolled in the Study

\begin{tabular}{ll}
\hline Age (years) & $68.3 \pm 8.10$ \\
Sex (male/female) & $7 / 3$ \\
Body weight $(\mathrm{kg})$ & $59.5 \pm 12.3$ \\
BMI $\left(\mathrm{kg} / \mathrm{m}^{2}\right)$ & $23.9 \pm 5.12$ \\
\hline Duration of diabetes (years) & $5.5(4-7.8)$ \\
\hline HbA1c (\%) & $7.7 \pm 0.52$ \\
Random plasma glucose (mg/dL) & $152 \pm 51$ \\
Random serum CPR (ng/mL) & $2.6(1.6-3.0)$ \\
Baseline 1,5-AG ( $\mu \mathrm{g} / \mathrm{mL})$ & $5.5 \pm 2.0$ \\
eGFR (mL/min/1.73 $\left.\mathrm{m}^{2}\right)$ & $73 \pm 10$ \\
Dyslipidemia (n) & 4 \\
\hline Hypertension (n) & 3 \\
Anti-hyperglycemic drugs & \\
\hline Metformin (n) & 8 \\
Alpha-glucosidase inhibitor (n) & 1 \\
\hline Pioglitazone (n) & 5 \\
Dipeptidyl peptidase-4 inhibitor (n) & 4 \\
\hline
\end{tabular}

Data are expressed as mean \pm standard deviation. BMI: body mass index; HbA1c: glycated hemoglobin; CPR: C-peptide immunoreactivity; 1,5-AG: 1,5-anhydroglucitol; eGFR: estimated glomerular filtration rate. with the HbA1c level, the post-challenge plasma glucose level was more strongly associated with the carotid intima-media thickness (IMT) after an oral glucose tolerance test [15]. Esposito et al reported that compared with glyburide, repaglinide better suppressed the progression of the carotid IMT in patients with type 2 diabetes and noted that a reduction in carotid IMT was associated with changes in the postprandial, but not fasting glucose level [16]. Yamazaki et al reported that in patients with type 2 diabetes, repaglinide reduced the mean amplitude of glycemic excursions, an index of daily blood glucose variability, and improved the urinary level of 8-hydoroxydeoxyguanosine, an oxidative stress marker, when compared with glimepiride [17]. Although this study suggests that an improvement in postprandial glucose levels is important for reducing oxidative stresses, the serum 1,5-AG level did not change after switching from glimepiride to repaglinide; this finding contradicts our study. This discrepancy can be attributed to differences in the patient background factors, such as the baseline HbAlc level, duration of diabetes mellitus or dosages of concomitant drugs.

Postprandial hyperglycemia is considered an important therapeutic target in the prevention of atherosclerosis. Recently, we reported the association of a lower 1,5-AG level with de novo coronary artery disease in both patients with and without type 2 diabetes and with adverse clinical events after percutaneous coronary intervention [18, 19]. These findings suggest that $1,5-\mathrm{AG}$ is a useful surrogate, predictive biomarker for adverse cardiac events. In addition, a recent meta-analysis revealed the association of severe hypoglycemia with cardiovascular events [20]. Interestingly, Haneda et al reported that sulfonylurea is a major cause of drug-induced hypoglycemia at a high-volume emergency center [21]; consistent with this, repaglinide was found to cause fewer hypoglycemic events when compared with glibenclamide, a common sulfonylurea drug [22]. Therefore, glinide drugs, such as repaglinide, could potentially be used to reduce postprandial hyperglycemia and confer a relatively low risk 

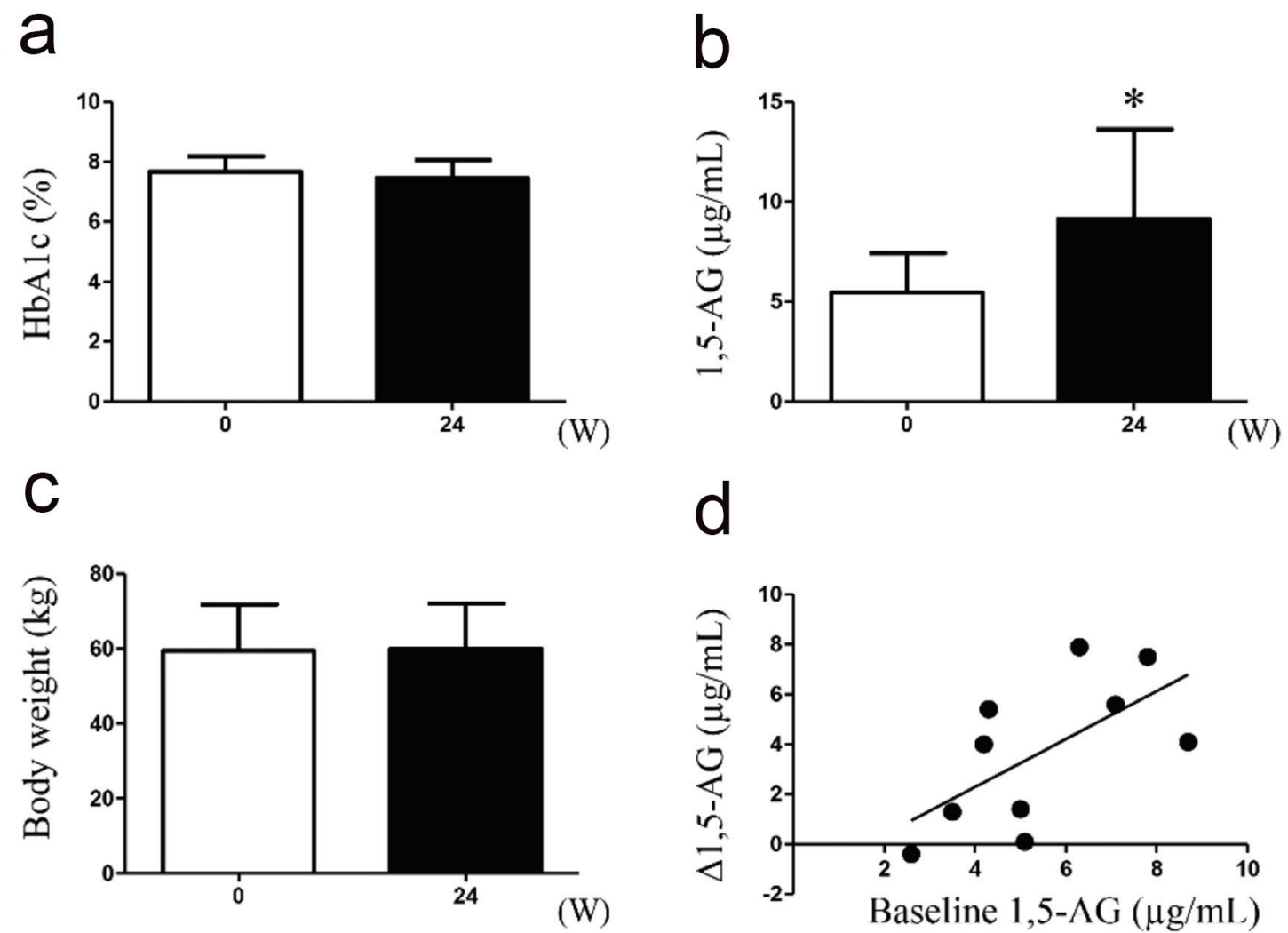

Figure 3. Measurement of oxidative stress markers in urine samples collected during treatment with either glimepiride or repaglinide. (a) Glycated hemoglobin (HbA1c); (b) 1,5-anhydroglucitol (1,5-AG); (c) body weight; (d) correlation between the baseline 1.5-AG and $\Delta 1.5-A G$ are indicated $(n=10) .{ }^{*} P<0.05$.

of severe hypoglycemia when compared with sulfonylurea drugs. Although a nationwide observational study revealed equivalent reductions in cardiovascular events with the use of repaglinide and metformin [23], no prospective clinical study has clearly evaluated the prevention of cardiovascular events in patients with type 2 diabetes. Therefore, further investigation is warranted to clarify the significance of improved postprandial hyperglycemia following repaglinide treatment in patients with type 2 diabetes.

The present study has some limitations. First, the study featured a single-arm design and small number of patients. A larger sample size and/or cross-over design should be applied in the future. Second, we did not completely control for the participants' lifestyle factors. Also, the switch between the antihyperglycemic drugs might have possibly affected the patients' functional or psychological states. Third, the glimepiride and repaglinide dosages differed among the patients; the baseline antihyperglycemic agents used by each patient also differed. This factor might have affected the glycemic control and, therefore, the study results. Lastly, we instructed diet therapy for all patients; however, the observance of diet therapy during this study affected results.

In conclusion, our findings indicate that compared with glimepiride, repaglinide improves the level of 1,5-AG, a marker for postprandial hyperglycemia, in patients with type 2 diabetes. Repaglinide may be a useful therapeutic option for treating postprandial hyperglycemia.

\section{Conflict of Interest}

The authors declare that they have no conflict of interest.

\section{Financial Support}

None.

\section{References}

1. Patel KL. Impact of tight glucose control on postoperative infection rates and wound healing in cardiac surgery patients. J Wound Ostomy Continence Nurs. 2008;35(4):397-404; quiz 405-396.

2. Tominaga M, Eguchi H, Manaka H, Igarashi K, Kato T, Sekikawa A. Impaired glucose tolerance is a risk factor for cardiovascular disease, but not impaired fasting glucose. The Funagata Diabetes Study. Diabetes Care. 1999;22(6):920-924.

3. Nakagami T, the DECODA Study Group. Hyperglycaemia and mortality from all causes and from cardiovascu- 
lar disease in five populations of Asian origin. Diabetologia. 2004;47(3):385-394.

4. Ceriello A. Postprandial hyperglycemia and diabetes complications: is it time to treat? Diabetes. 2005;54(1):17.

5. Ceriello A. The post-prandial state and cardiovascular disease: relevance to diabetes mellitus. Diabetes Metab Res Rev. 2000;16(2):125-132.

6. Monnier L, Lapinski H, Colette C. Contributions of fasting and postprandial plasma glucose increments to the overall diurnal hyperglycemia of type 2 diabetic patients: variations with increasing levels of $\mathrm{HbA}(1 \mathrm{c})$. Diabetes Care. 2003;26(3):881-885.

7. Ando K, Nishimura R, Tsujino D, Seo C, Utsunomiya K. 24-hour glycemic variations in drug-naive patients with type 2 diabetes: a continuous glucose monitoring (CGM)based study. PLoS One. 2013;8(7):e71102.

8. Dungan KM, Buse JB, Largay J, Kelly MM, Button EA, Kato S, Wittlin S. 1,5-anhydroglucitol and postprandial hyperglycemia as measured by continuous glucose monitoring system in moderately controlled patients with diabetes. Diabetes Care. 2006;29(6):1214-1219.

9. Yamanouchi T, Moromizato H, Shinohara T, Minoda S, Miyashita H, Akaoka I. Estimation of plasma glucose fluctuation with a combination test of hemoglobin A1c and 1,5-anhydroglucitol. Metabolism. 1992;41(8):862867.

10. Nonaka K, Kakikawa T, Sato A, Okuyama K, Fujimoto G, Kato N, Suzuki H, et al. Efficacy and safety of sitagliptin monotherapy in Japanese patients with type 2 diabetes. Diabetes Res Clin Pract. 2008;79(2):291-298.

11. Marbury TC, Ruckle JL, Hatorp V, Andersen MP, Nielsen KK, Huang WC, Strange P. Pharmacokinetics of repaglinide in subjects with renal impairment. Clin Pharmacol Ther. 2000;67(1):7-15.

12. Haneda M, Noda M, Origasa H, Noto H, Yabe D, Fujita Y, Goto A, et al. Japanese Clinical Practice Guideline for Diabetes 2016. J Diabetes Investig. 2018;9(3):657-697.

13. Matsuo S, Imai E, Horio M, Yasuda Y, Tomita K, Nitta $\mathrm{K}$, Yamagata $\mathrm{K}$, et al. Revised equations for estimated GFR from serum creatinine in Japan. Am J Kidney Dis. 2009;53(6):982-992.

14. Kanda Y. Investigation of the freely available easy-touse software 'EZR' for medical statistics. Bone Marrow
Transplant. 2013;48(3):452-458.

15. Temelkova-Kurktschiev TS, Koehler C, Henkel E, Leonhardt W, Fuecker K, Hanefeld M. Postchallenge plasma glucose and glycemic spikes are more strongly associated with atherosclerosis than fasting glucose or HbAlc level. Diabetes Care. 2000;23(12):1830-1834.

16. Esposito K, Giugliano D, Nappo F, Marfella R, Campanian Postprandial Hyperglycemia Study G. Regression of carotid atherosclerosis by control of postprandial hyperglycemia in type 2 diabetes mellitus. Circulation. 2004;110(2):214-219.

17. Yamazaki M, Hasegawa G, Majima S, Mitsuhashi K, Fukuda T, Iwase H, Kadono M, et al. Effect of repaglinide versus glimepiride on daily blood glucose variability and changes in blood inflammatory and oxidative stress markers. Diabetol Metab Syndr. 2014;6:54.

18. Fujiwara T, Yoshida M, Akashi N, Yamada H, Tsukui T, Nakamura T, Sakakura K, et al. Lower 1,5-anhydroglucitol is associated with adverse clinical events after percutaneous coronary intervention. Heart Vessels. 2016;31(6):855-862.

19. Fujiwara T, Yoshida M, Yamada H, Tsukui T, Nakamura T, Sakakura K, Wada H, et al. Lower 1,5-anhydroglucitol is associated with denovo coronary artery disease in patients at high cardiovascular risk. Heart Vessels. 2015;30(4):469-476.

20. Goto A, Arah OA, Goto M, Terauchi Y, Noda M. Severe hypoglycaemia and cardiovascular disease: systematic review and meta-analysis with bias analysis. BMJ. 2013;347:f4533.

21. Haneda M, Morikawa A. Which hypoglycaemic agents to use in type 2 diabetic subjects with CKD and how? Nephrol Dial Transplant. 2009;24(2):338-341.

22. Papa G, Fedele V, Rizzo MR, Fioravanti M, Leotta C, Solerte SB, Purrello F, et al. Safety of type 2 diabetes treatment with repaglinide compared with glibenclamide in elderly people: A randomized, open-label, two-period, cross-over trial. Diabetes Care. 2006;29(8):1918-1920.

23. Schramm TK, Gislason GH, Vaag A, Rasmussen JN, Folke F, Hansen ML, Fosbol EL, et al. Mortality and cardiovascular risk associated with different insulin secretagogues compared with metformin in type 2 diabetes, with or without a previous myocardial infarction: a nationwide study. Eur Heart J. 2011;32(15):1900-1908. 